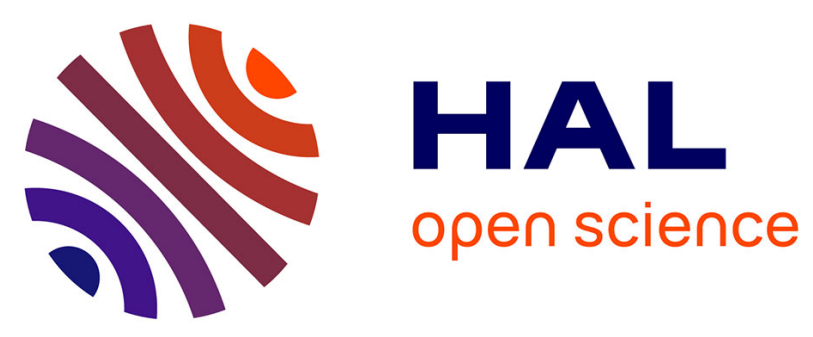

\title{
Spatio-Temporal Shape Analysis of Cross-Sectional Data for Detection of Early Changes in Neurodegenerative Disease
}

Claire Cury, Marc M Lorenzi, David M Cash, Jennifer M Nicholas, Alexandre M Routier, Jonathan Rohrer, Sebastien M Ourselin, Stanley M Durrleman, Marc M Modat

\section{To cite this version:}

Claire Cury, Marc M Lorenzi, David M Cash, Jennifer M Nicholas, Alexandre M Routier, et al.. Spatio-Temporal Shape Analysis of Cross-Sectional Data for Detection of Early Changes in Neurodegenerative Disease. SeSAMI 2016 - First International Workshop Spectral and Shape Analysis in Medical Imaging, Sep 2016, Athens, Greece. pp.63 - 75, 10.1007/978-3-319-51237-2_6 . hal01440061

\section{HAL Id: hal-01440061 \\ https://hal.inria.fr/hal-01440061}

Submitted on 18 Jan 2017

HAL is a multi-disciplinary open access archive for the deposit and dissemination of scientific research documents, whether they are published or not. The documents may come from teaching and research institutions in France or abroad, or from public or private research centers.
L'archive ouverte pluridisciplinaire $\mathbf{H A L}$, est destinée au dépôt et à la diffusion de documents scientifiques de niveau recherche, publiés ou non, émanant des établissements d'enseignement et de recherche français ou étrangers, des laboratoires publics ou privés. 


\title{
Spatio-temporal shape analysis of cross-sectional data for detection of early changes in neurodegenerative disease
}

\author{
Claire Cury ${ }^{1,2}$, Marco Lorenzi ${ }^{1}$, David Cash ${ }^{1,2}$, Jennifer M Nicholas ${ }^{2,3}$, \\ Alexandre Routier ${ }^{4}$, Jonathan Rohrer ${ }^{2}$, Sebastien Ourselin ${ }^{1,2}$, Stanley \\ Durrleman $^{4}$, and Marc Modat ${ }^{1,2}$ \\ 1 Translational Imaging Group, Centre for Medical Image Computing, Medical \\ Physics and Biomedical Engineering Department, University College London, London \\ NW1 2HE, United Kingdom \\ 2 Dementia Research Centre, Institute of Neurology, University College London, \\ London WC1N 3BG, United Kingdom \\ 3 Department of Medical Statistics, London School of Hygiene \& Tropical Medicine, \\ London, United Kingdom \\ ${ }^{4}$ Inria Aramis project-team Centre Paris-Rocquencourt, Inserm U 1127, CNRS \\ UMR 7225, Sorbonne Universits, UPMC Univ Paris 06 UMR S 1127, Institut du \\ Cerveau et de la Moelle pinire, ICM, F-75013, Paris, France
}

\begin{abstract}
The detection of pathological changes in neurodegenerative diseases that occur before clinical onset would be of great value for identifying suitable subjects and assessing drug efficacy in trials aimed at preventing or slowing onset. Using MRI derived volumetric information, researchers have been able to detect significant differences between patients in the presymptomatic phase of neurodegenerative diseases and healthy controls. However, volumetric studies provide only a summary representation of complex morphological changes. Shape analysis has already been successfully applied to model pathological features in neurodegeneration and represents a valuable instrument to model presymptomatic anatomical changes occurring in specific brain regions.

In this study we propose a computational framework to model groupwise spatio-temporal shape differences, and to statistically evaluate the effects of time and pathological components on the modeled variability. The proposed approach leverages the geodesic regression framework based on varifolds, and models the spatio-temporal shape variability via dimensionality reduction of the subject-specific "residual" transformations normalised in a common reference frame through parallel transport. The proposed approach is applied to patients with genetic variants of fronto-temporal dementia, and shows that shape differences in the posterior part of the thalamus can be observed several years before the appearance of clinical symptoms.
\end{abstract}

Keywords: shape, thalamus, spatio-temporal geodesic regression, FTD, parallel transport 


\section{Introduction}

The hallmark of neuro-degenerative diseases such as Alzheimer's disease (AD) or frontotemporal dementia (FTD) are the progressive clinical symptoms that include dementia, memory loss, and changes in behaviour. However, there is also evidence of pathological changes occurring much earlier than the onset of these clinical symptoms. This presymptomatic phase of the disease can last more than a decade. Reliably detecting these early changes in presymptomatic individuals could provide the roadmap to improved prevention of these diseases. These findings would not only provide better understanding of the underlying mechanisms of the disease, but they would also result in improved identification of at-risk individuals that would be suitable for potential therapies that will halt or slow down the disease process. Accurate, reliable measurements of these changes could also be used to assess the efficacy of these therapies in secondary prevention clinical trials.

Autosomal dominant forms of dementias provide a reliable means of identifying presymptomatic individuals who are highly likely to develop the disease. The Dominantly Inherited Alzheimer Network (DIAN) and the Genetic FTD Initiative (GENFI) are examples of international studies of autosomal dominant forms of AD and FTD that are collecting multimodal neuroimaging, alongside other biomarkers with the objective of obtaining an improved understanding of the changes that are occuring during the presymptomatic phase of the disease. The structural MRI results from these studies $[2,19]$ have shown evidence of significant volume differences between carriers and non-carriers in numerous regions of the brain years before the expected onset of clinical symptoms.

While the neurodegenerative process has consistently resulted in downstream effects of regional volume, there may be even earlier, more sensitive information, encoded in the shape of the structure. An important aspect of shape analysis is their description. Different ways to describe shape have been proposed. The use of M-Representation [18] allows atrophy measurements and enables the separation of the thickness of a shape from its positioning. However, this representation is sensitive to topological changes. Spherical harmonic decompositions $\underline{23]}$ are often used for their simplicity in parametrisation since only a few parameters are required to describe a shape. However, the interpretation of the parameters, and differences between them, is not intuitive. For the current study we represent shapes using varifolds [4], which have the same properties as the current representation [25]. They are robust to varying topologies, do not require point to point correspondences, and embed the shapes in a vector space, which facilitate the interpretation of results.

In this paper the methodology is based on the framework of the Large Deformation Diffeomorphic Metric Mapping (LDDMM) [24,1,12], providing a shape space, there is a continum between all shapes of the population under study. we develop a for spatio-temporal shape analysis in order to qualify and quantify early shape changes between subjects with and without genetic variants of FTD. We first compute an average spatio-temporal trajectory of the thalamic shapes segmented from a population of 211 individuals $[8,11]$. Second, we 
compute the subject-specific "residual" trajectories with respect to the modelled progression, and we subsequently spatio-temporaly normalise them in the baseline reference frame through parallel transport $[26]$. We finally model the spatio-temporal variability encoded in the "residuals" through kernel PCA. The individual projections in the latent space are statistically analysed via random effect models to investigate significant effects of time and group-wise differences in the encoded longitudinal variability. In the literature we can find different longitudinal shape analysis answering different questions. The work presented in Lorenzi et al. (2010) [14] measures brain atrophy for each subject using follow up scans, and the method is not based on the LDDMM framework. In Datar et al. [6] they used a shape representation based on point to point correspondences and tend to model population trend. In Lorenzi et al. (2015) [16] they estimate the anatomical age of a new subject regarding to a normal aging longitudinal model based on stationary vector fields. The method aims to estimate from brain images the contribution of aging and pathology. Here the aim of the method is to detect shape differences due to pathology along the time.

\section{Method}

We indicate with $\left\{\left(S_{i}, t_{i}\right)\right\}_{i \in\{0, N-1\}}$ a set of $N$ shapes associated with a corresponding time point $t_{i}$. With analogy to classical random-effect-modelling approaches, we assume that each shape is a random realisation of a common underlying spatio-temporal process $\phi(t)$ :

$$
S_{i}=\rho_{i}\left(\phi\left(t_{i}, B_{0}\right)\right)+\epsilon_{i}
$$

where $B_{0}$ is a common reference frame (typically the baseline image), and $\rho_{i}$ is a subject-specific "residual" accounting for individual variations, this is the diffeomorphic deformation linking the mesh of the shape $S_{i}$ to the corresponding time point $t_{i}$ of the common trajectory. We also assume that $\epsilon_{i}$ is Gaussian random distributed noise. In order to identify group-wise differences between the spatio-temporal trajectory changes, we propose a statistical framework to model and compare the subjects-specific "residuals" $\rho_{i}$. This is a challenging problem, since the $\rho_{i}$ are defined at different time points, and therefore cannot be directly compared in a common anatomical framework. Moreover, the optimisation of the functional for the simultaneous estimation of group-wise trajectory and random effects is not trivial, and would ultimately result in highly expensive numerical schemes. For this reason, we propose to simplify the optimisation problem by introducing an efficient numerical framework composed of three steps illustrated in Figure 1. (i) First we assume that the "residuals" $\rho_{i}$ are fixed, and estimate the common trajectory $\phi(t)$. (ii) Second, given the modelled trajectory $\phi$, we estimate the random effects $\rho_{i}$ through non-linear registration between the trajectory point $\phi\left(B_{0}, t_{i}\right)$ and $S_{i}$. (iii) Third, after normalising the random effects in the common baseline reference space $B_{0}$, we evaluate group-wise differences and time dependencies. This is achieved through dimensionality reduction and subsequent univariate analysis on the reduced projections. 


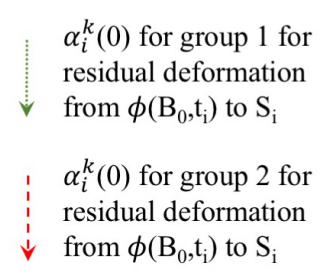

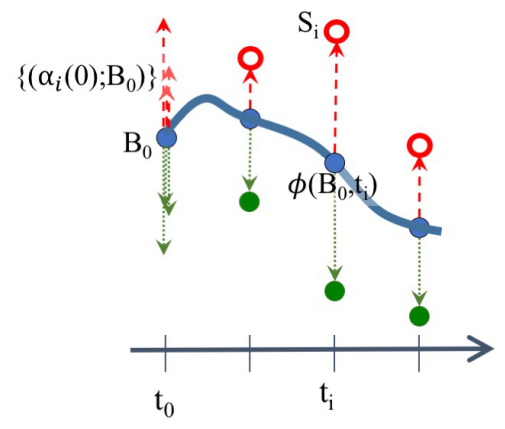

Fig. 1. Proposed framework. An average continuous shape trajectory is first computed from a population (composed by 2 groups of individuals). Second the "residual" between each shape and the trajectory is computed before being parallel transported to a common time point $\left(B_{0}\right)$ for statistical analysis.

The proposed framework relies on the mathematical setting of the Large Diffeomorphic Deformation Metric Mapping (LDDMM) framework and the varifold representation of shapes. This choice allows a mathematically consistent definition of (i) the spatio-temporal regression, (ii) the $\rho_{i}$ deformations estimation, and (iii) the normalisation of the initial momentum of $\rho_{i}$ through parallel transport.

\subsection{Large Diffeomorphic Deformation Metric Mapping and varifold representation}

To quantify differences between shapes, we use diffeomorphic deformations that provide smooth mappings between spaces and are invertible with smooth inverses. The LDDMM framework $[24, \underline{1]}$ is a mathematical and algorithmic framework based on flows of diffeomorphisms, which allows comparing anatomical shapes as well as performing statistics. The framework used in this paper is in fact a discrete parametrization of the LDDMM framework, as proposed in Durrleman et al. 2011 [10], based on a finite set of control points. The control points can be located anywhere not necessarily at the shape vertices, and they are independent of the shapes to be matched. They define a potentially infinitedimensional basis of the parametrization of the deformation. The vector attached to them define the weights of the decomposition of a given deformations onto this basis, these vectors are called momentum vectors. The position of the control points and the momentum vectors attached to them need to be optimized. The optimisation of the control points concentrate them in the most variable parts of the object to match as shown in Durrleman et al. (2013) [7].

Deformation maps $\varphi_{v}: \mathbb{R}^{3} \rightarrow \mathbb{R}^{3}$ are built by integrating time-varying vector fields $\left(v_{t}\right)_{0 \leq t \leq 1}$, such that each $v(\cdot, t)$ belongs to a Reproducing Kernel 
Hilbert Space (RKHS) $V$ with kernel $K_{V}$, we used a Gaussian kernel:

$$
k_{V}(x, y)=\exp \left(\frac{-|x-y|^{2}}{\lambda^{2}}\right) \operatorname{Id}, \text { for all } \operatorname{points}(x, y)
$$

with Id the identity matrix, and $\lambda$ a scale factor which determines the size of the kernel and therefore the degree of smoothness of the deformations. We define $\varphi_{v}(x)=\phi_{v}(x, 1)$ as the diffeomorphism induced by $v(x, t)$ where $\phi_{v}(x, 1)$ is the unique solution of the differential equation:

$$
\frac{d \phi_{v}}{d t}(x, t)=v\left(\phi_{v}(x, t), t\right), \forall t \in[0,1] \text { with } \phi_{v}(x, 0)=x, \forall x \in \mathbb{R}^{3} .
$$

Velocity fields $\left(v_{t}\right)$ are controlled via an energy functional $\int_{0}^{1}\|v(\cdot, t)\|_{V}^{2} d t$, where $\|\cdot\|_{V}$ is a Hilbert norm defined on vector fields of $\mathbb{R}^{3}$, which penalises nonregularity. This energy is used as a regularity term in the matching functional. Matching two shapes $S$ and $T$ needs the estimation of an optimal deformation map $\phi: \mathbb{R}^{3} \rightarrow \mathbb{R}^{3}$ such that $\phi(S)$ is close to $T$ by optimising a functional

$$
E\left(\phi_{v}\right)=d\left(\varphi_{v}(S), T\right)^{2}+\gamma \int_{0}^{1}\|v(\cdot, t)\|_{V}^{2} d t
$$

where $\gamma$ balances between the regularity of $\phi$ and the spatial proximity $d$, a similarity measure between the varifold representation of $\phi(S)$ and $T$.

In a discrete setting, the vector fields $v(x, t)$ corresponding to optimal maps are expressed as combinations of spline fields which involve the reproducing kernel $K_{V}$ of the space $V$ :

$$
v(x, t)=\sum_{p=1}^{n} K_{V}\left(x, x_{p}(t)\right) \alpha_{p}(t),
$$

where $x_{p}(t)=\phi_{v}\left(x_{p}, t\right)$ are the trajectories of control points $x_{p}$, in our case the control points are regularly spaced on a 3D grid containing the mesh of the subject $S$. The spacing between the control points is defined from the size of the kernel $K_{V} \cdot \alpha_{p}(t) \in \mathbb{R}^{3}$ are time-dependent vectors called momentum vectors. The optimal trajectories between shapes $S$ and $T$, satisfy the geodesic equations for a given metric on the set of control points such as the varifolds [4]. As a result the full deformation can be encoded by the vector of initial momentum vectors $\boldsymbol{\alpha}(0)=\left\{\alpha_{p}(0)\right\}_{1<p<n}$ located at the points $\left\{x_{p}\right\}_{1<p<n}$. This allows to analyse the set of deformation maps from a given template to the observed shapes by performing statistics on the initial momentum vectors located on the template shape. The process of generating back any deformation maps from initial conditions $\left(x_{p}(0), \alpha_{p}(0)\right)$, i.e. integrating the geodesic equations, is called geodesic shooting or exponential map and is noted $\exp _{x_{p}(0)}\left(\alpha_{p}(0)\right)$.

As said in the introduction we used the varifolds to represent our shapes [4]. This is the non oriented version of the representation with currents which is very efficient to model a large range of shapes. To represent a shape $\mathrm{S}$ as a 
varifold, the shape space is embedded into a Reproducing Kernel Hilbert Space (RKHS), where it is encoded using a set of non-oriented unit normals attached on each vertices of the shape. As for the current representation, this kernelbased embedding allows to define a proper distance between different embedded shapes. Here the studied shape is the thalamus, which has an ovoid shape, so currents or varifolds could have been used, the orientation of the normals on the shape are not an issue.

\subsection{Proposed framework}

The computation of the spatio-temporal regression $[8]$ requires its initialisation to a baseline shape $B_{0}=\left\{x_{p}\right\}_{p=1, \ldots, N_{B_{0}}}$, where $N_{B_{0}}$ denotes the number of control points defined on the shape $B_{0}$. To avoid any bias of the geodesic regression towards an initial subject selected as baseline, we estimate the baseline from the 10 youngest subjects regarding the temporal axis, so subjects with the smallest $t_{i}$. The baseline is estimated by computing iteratively the centroid of those subjects in the space of deformations, using the diffeomorphic Iterative Centroid method [5], based on the LDDMM framework.

The spatio-temporal regression of the set of shapes $\left\{\left(S_{i}, t_{i}\right)\right\}_{i \in\{0, N\}}$ is implemented in the Deformetrica ${ }^{5}$ software $[9,21]$. The method requires the discretisation of the temporal axes using $T$ time points, a value specified by the user. The new set of data, used for the regression, is $\left\{\left(S_{i}, t\right)\right\}_{i \in\{0, N\}, t \in\{0, T\}}$, where

$$
t=\underset{t}{\operatorname{argmin}}\left\{\left\|t-t_{i}\right\|, \forall t \in\{0, \ldots, T\}\right\} .
$$

The method computes a geodesic starting at position $B_{0}$ at time $t=0$, and moving to positions $\phi\left(B_{0}, t\right) \forall t \in\{0, T\}$, following the differential equation seen previously, and minimising the discrepancy between the model at time $t$ (i.e. $\left.\phi\left(B_{0}, t\right)\right)$ and the actual observation $S_{i}$ :

$$
E(\phi)=\sum_{t} d\left(\phi\left(B_{0}, t\right), S_{i}\right)^{2}+\gamma\left\|\left|v_{0}^{\phi} \|\right|_{V^{\phi}}^{2},\right.
$$

with $v^{\phi}$ the time-varying velocity vector field that belongs to the RKHS $V^{\phi}$ determined by the Gaussian Kernel $K^{\phi}$. The initial momentum vector $\alpha(0)$ is defined on the control points of the baseline shape $B_{0}$ and fully encodes the geodesic regression.

Then from the spatio temporal trajectory, we compute the "residuals" deformations $\rho_{i}$ between every observation and the spatio-temporal average shape by estimating the geodesic between $\phi\left(B_{0}, t_{i}\right)$ and $\left\{S_{i}, t_{i}\right\}$, using the matching equation seen in section 2.1. We then obtain a set of momenta $\left\{\phi\left(B_{0}, t\right) ; \alpha_{i}(0)\right\}_{t \in\{0 ; T\}}$ that encodes the deformations $\rho_{i}$ from the spatio-temporal regression to all subjects. To make this set of momenta comparable, we need to define them in the same space.

\footnotetext{
${ }^{5}$ http://www.deformetrica.org/
} 
We transport all momenta into the baseline space of $B_{0}=\phi\left(B_{0}, 0\right)$, using a parallel transport method based on Jacobi fields as introduced in [26]. Parallel transporting a vector along a curve (the computed trajectory parametrised by $\left\{B_{0} ; \alpha(0)\right\}$ here) consists in translating it across the tangent spaces to the curve by preserving its parallelism, according to a given connection (the Levi-Civita connection in LDDMM). The vector is parallel transported along the curve if the connection is null, for all steps along the curve [15]. We chose to use Jacobi field instead of the Schild's Ladder method [13], to avoid the cumulative errors and the excessive computation time due to the computation of Riemannian Logarithms in the LDDMM framework, required for the Schild's Ladder. Those errors would have been different from a subject to an other, since they all are at different time points of the trajectory, some of them have to travel more than the others. To transport a vector $\eta$ from a time $t$ to the time $t_{0}=0$, along the geodesic $\gamma$, the Jacobian field is defined as:

$$
J_{\gamma(t)}\left(0,-\alpha_{i}(t), \eta\right)=\frac{\partial}{\partial \epsilon} \exp _{\gamma(t)}\left(1 / T\left(-\alpha_{i}(t)+\epsilon \eta\right)\right) .
$$

The geodesic $\gamma(t)$ is in the direction $-\alpha_{i}(t)$, and $\eta$ is an initial momentum vector as the $\left\{\alpha_{i}(0)\right\}$ computed above, is tangent to the geodesic $\gamma$ at the time point $t$.

We then have $\mathrm{N}$ vectors of size $3 \times N_{B_{0}}$ defined in $B_{0}$. This information is reduced using a Kernel Principal Component Analysis (K-PCA) [22], which is the non-linear version of the standard Principal Component Analysis (PCA). The covariance matrix is defined as:

$$
\Gamma_{i, j}^{V^{\phi}}=\left(\alpha_{i}-\bar{\alpha}\right) K_{V^{\phi}}\left(\alpha_{j}-\bar{\alpha}\right)
$$

where $V^{n}$ are the eigenvectors of the matrix $\Gamma^{V}$, with $K_{V^{\phi}}$ the kernel of the space of deformations used for the computation of the spatio-temporal regression. The $n$-th mode of variation is defined as

$$
m^{\alpha}=\bar{\alpha}+\sum_{i} V_{i}^{n}\left(\alpha^{i}-\bar{\alpha}\right) .
$$

\section{$3 \quad$ Data and experiments}

We applied the proposed framework to the GENFI database, using the thalamus as our structure of interest. GENFI is a multi-centre study in which participants come from families known to carry a pathogenic mutation in one of three genes that are the most common cause of genetic FTD: microtubule-associated protein tau (MAPT), progranulin (GRN) and an expansion of the open reading frame 72 in Chromosome 9 (C9orf72). The thalamus is an intriguing candidate for this analysis, as Rohrer et al. [19] reported volumetric differences in the thalamus 5 years before expected age at onset. In this paper we used 211 participants, 113 mutation carriers and 98 non-carriers. All participants have a T1-weighted (T1w) MRI and an expected years to symptom onset (EYO). Table 1 shows the demographics of the study participants used in this analysis. 
Table 1. Data demographics

\begin{tabular}{lll} 
& $\begin{array}{l}\text { Non-carriers } \\
\mathrm{n}=98\end{array}$ & $\begin{array}{l}\text { Mutation carriers } \\
\mathrm{n}=113\end{array}$ \\
\hline Males & 59 & 56 \\
Asymptomatic & 98 & 76 \\
Age in years (med (IQR)) & $50.2(36.6-62.1)$ & $52.7(41.1-62.7)$ \\
Years from expected onset: & & \\
$\leq-20$ years & 30 & 21 \\
$-20 \leq$ years $\leq-10$ & 16 & 21 \\
$-10 \leq$ years $<0$ & 23 & 22 \\
$0 \leq$ years & 29 & 49 \\
\hline
\end{tabular}

Before running the spatio-temporal regression, we first ran the parcellation of the $\mathrm{T} 1 \mathrm{w}[3]$ to extract afterwards the meshes of the structure of interest. Second, we rigidly and affinely aligned the $\mathrm{T} 1 \mathrm{w}$ brain images to a groupwise space before rigidly refining the alignment of the thalamus parcellations [17]. Next, we extracted the meshes corresponding to the left thalamus, including around 2,300 vertices for 441 control points. We have 211 left thalamus meshes associated with the EYO of the subject and the indication if the subject is mutation carrier (MC) or not. For the regression we used 30 time points, which corresponds approximatively to one time point every two years. The space of deformations $V$ was defined using a $11 \mathrm{~mm}$ width kernel, which corresponds to half of the length of a thalamus, and a $5 \mathrm{~mm}$ width for he space of varifolds.

Similarly to the volumetric analysis performed by Rohrer et al. [19], we use a mixed effect model to study the shape difference between the healthy subjects and the MC. The eigenvectors computed from the principal component analysis of the "residual" deformations transported in the baseline shape are used as variables and the fixed effects predictors of interest are mutation carrier status, EYO and interaction between mutation carrier status and EYO. To allow for non-linear change in thalamus volume or shape the model includes a term for $\mathrm{EYO}^{2}$ and the interaction between mutation carrier status and $\mathrm{EYO}^{2}$. A random intercept for family allows values of the marker to be correlated between family members.

We did a Wald test on the first principal component, which represents $20.4 \%$ of the variability of the residual deformations, another on the second component which represents $11.3 \%$ of the variability, and on the third component $(10.8 \%$ of the variability). We were also interested by the interaction of these components, so we conducted joint Wald test on the two first components $(31.7 \%$ of the variability), and on the three first components ( $42.5 \%$ of the variability). For each analysis, further Wald tests were conducted every 5 years as in the volumetric analysis [19] to assess how long before the expected onset we could detect evidence for differences between mutation carriers and controls.

The results depend on the metric used for computing the trajectory and for computing the deformations $\rho_{i}$. The metric depend on the space of deformation 
used, which is defined by the kernel $K_{V}$ used. Choosing a bigger kernel size for the deformation space would lead to different trajectory, with less differences, then to different results. Choosing a smaller kernel size would give a more variable trajectory, leading to different results. The size of the kernel $K_{V}$ of the space of deformations were chosen to be half of the thalamus size.

\section{Results}

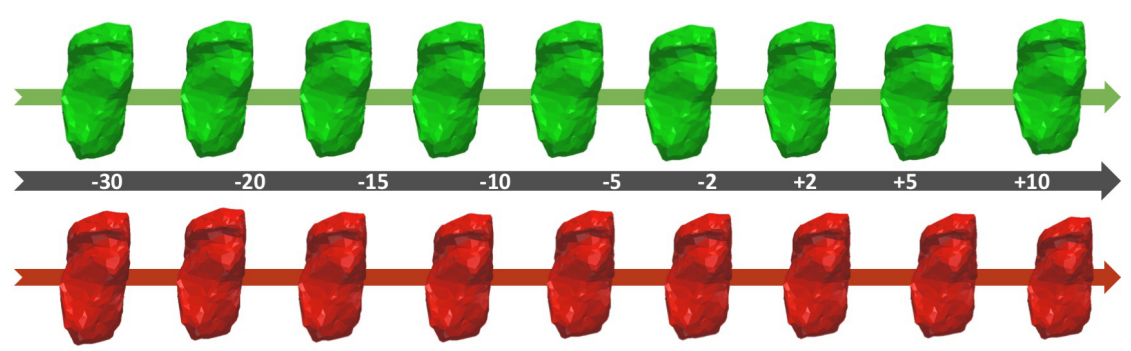

Fig. 2. Geodesic trajectories of the left thalamus, for the control group (first row, green) and for the mutation carrier group (bottom row, red). The figure shows a bottom view of the thalamus. The middle grey line indicates the expected years to onset (EYO) for both trajectories.

Results from the analysis of the left thalamus shapes, comparing mutation carriers and controls, including up to 3 principal components are shown in table 2. To visualise the shape differences between the two groups, we computed two trajectories with the same parameters and the same baseline. One for the mutation carrier group, and one for the control group. Figure 2 shows these trajectories, we can see that the trajectories are indeed similar at the beginning, they progressively differ from each others. At the end of the trajectories, the shapes are clearly different.

Table 2. p-values for differences in the shape of the left thalamus between groups, by expected years to symptom onset and per principal component (PC)

\begin{tabular}{l|rrrrrrcc} 
PCs (captured var.) $\backslash$ EYO & -25 & -20 & -15 & -10 & -5 & 0 & +5 & +10 \\
\hline PC 1 $(20.4 \%)$ & 0.70 & 0.94 & 0.60 & 0.16 & $8 \mathrm{e}-3$ & $<1 \mathrm{e}-3<1 \mathrm{e}-3$ & $<1 \mathrm{e}-3$ \\
PC $2(11.3 \%)$ & 0.42 & 0.26 & 0.16 & 0.08 & 0.03 & 0.01 & 0.01 & 0.03 \\
PC $3(10.8 \%)$ & 0.29 & 0.13 & 0.08 & 0.06 & 0.06 & 0.08 & 0.25 & 0.62 \\
\hline PC $1+2(31.7 \%)$ & 0.78 & 0.80 & 0.77 & 0.45 & 0.07 & $1 \mathrm{e}-3$ & $<1 \mathrm{e}-3$ & $<1 \mathrm{e}-3$ \\
PC $1+2+3(42.5 \%)$ & 0.76 & 0.49 & 0.23 & 0.05 & $2 \mathrm{e}-3$ & $<1 \mathrm{e}-3<1 \mathrm{e}-3$ & $<1 \mathrm{e}-3$
\end{tabular}




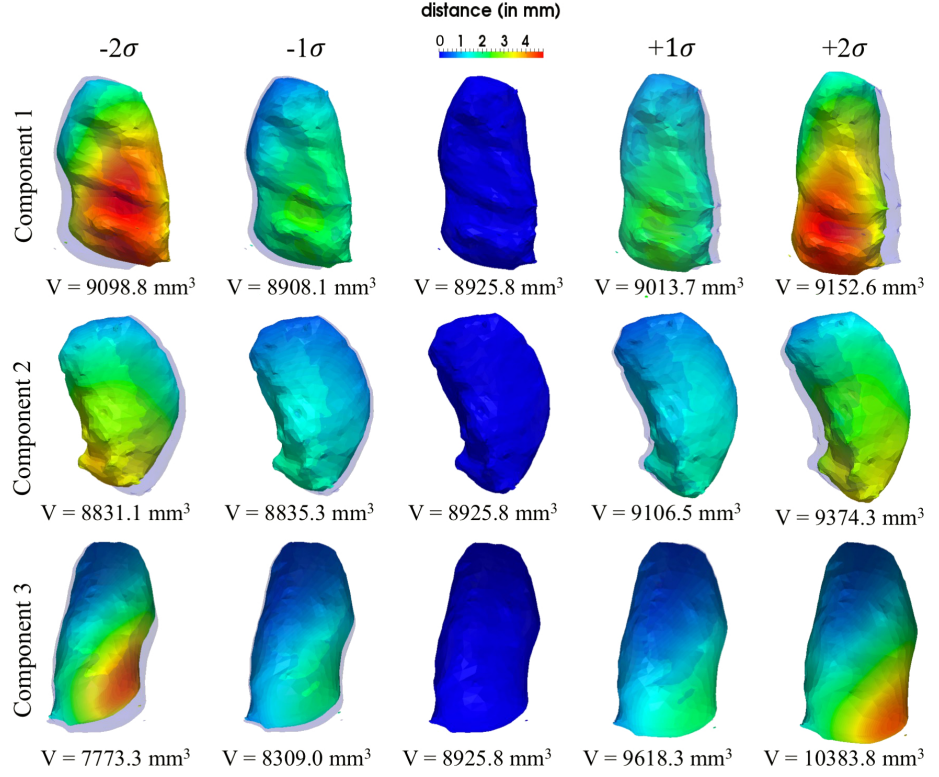

Fig. 3. First three modes of variation captured from the "residuals" between each individual and the average spatio-temporal regression on the whole population. Whereas the second and third components exhibit correlation with the thalamus volume, the first component exhibits a constant volume. It can be observed that the captured variations is located in the posterior part of the thalamus.

On table 2 the first two principal components capture individually significant shape differences 5 years before onset, which is not the case for the third component. The combination of the three components increases the significance of the differences between the two groups and we can then observe significant shape differences 10 years before the expected symptom onset. The results are not corrected for multiple comparison to have a head to head correspondance with the Rohrer et al. [19] volumetric study. We also have strong a-priori expectation that there would be real differences between mutation carriers and no-carriers, furthermore multiple comparison would provide protection against type I error (false positive), while increasing the probability of making type II error (false negative) [20]. Figure 3 shows the first three modes of variation of the deformations, with the information of volume for each component. It can be observed that the first component, which captures approximatively $20 \%$ of the variability, does not embed volumetric information. The volume information is however captured by the second and the third component. The first three modes of variation show deformation mainly located in the posterior part of the thalamus, which dorso-posterior part is connected to the limbic system, implicated in frontotemporal dementia. 


\section{Conclusion}

Using the proposed framework for spatio-temporal shape analysis, we have been able to detect shape differences 10 years before the expected onset of clinical symptoms associated with frontotemporal dementia. Using volumetric information alone, Rohrer et al. [19] were able to detect statistical differences 5 years prior to clinical onset using the same statistical analysis method. Not only does the proposed framework provide the potential for more sensitivity, but it also allows for better spatial localisation of the changes within the structure. As shown by the first mode of variation captured from the data, there are differences between the groups that are not captured by the volumetric information. These differences are located in the posterior part of the thalamus. In this study, we focused on the left thalamus. In the future we will investigate how much information can be extracted from other regions of interest that exhibited volumetric differences. We will also further enhance our framework to include longitudinal information with the aim to better capture the shape differences between the groups.

\section{Acknowledgements}

Claire Cury is supported by the EU-FP7 project VPH-DARE@IT (FP7-ICT2011-9-601055). Marco Lorenzi received funding from the EPSRC (EP/J020990/1). Jennifer Nicholas is supported by UK Medical Research Council (grant MR/M023664/1). David Cash is supported by grants from the Alzheimer Society(AS-PG-15-025), Alzheimers Research UK (ARUK-PG2014-1946) and Medical Research Council UK (MR/M023664/1). Stanley Durrleman has received funding from the program Investissements davenir ANR-10-IAIHU-06 and the European Unions Horizon 2020 research and innovation programme EuroPOND under grant agreement No 666992. Jonathan D. Rohrer is an MRC Clinician Scientist and has received funding from the NIHR Rare Diseases Translational Research Collaboration. Sebastien Ourselin receives funding from the EPSRC (EP/H046410/1, $\mathrm{EP} / \mathrm{K} 005278)$, the MRC (MR/J01107X/1), the NIHR Biomedical Research Unit (Dementia) at UCL and the National Institute for Health Research University College London Hospitals Biomedical Research Centre (NIHR BRC UCLH/UCL High Impact Initiative- BW.mn.BRC10269). Marc Modat is supported by the UCL Leonard Wolfson Experimental Neurology Centre (PR/ylr/18575) and Alzheimers Society UK (AS-PG-15-025). We would like to thank the participants and their families for taking part in the GENFI study.

\section{References}

1. Beg, M.F., Miller, M.I., Trouvé, A., Younes, L.: Computing large deformation metric mappings via geodesic flows of diffeomorphisms. International Journal of Computer Vision 61(2), 139-157 (Oct 2005) 
2. Benzinger, T.L.S., Blazey, T., Jack, C.R., et al.: Regional variability of imaging biomarkers in autosomal dominant Alzheimer's disease. Proceedings of the National Academy of Sciences 110(47), E4502-9 (Nov 2013)

3. Cardoso, M.J., Leung, K., Modat, M., Keihaninejad, S., Cash, D., Barnes, J., Fox, N.C., Ourselin, S., for ADNI: STEPS: Similarity and truth estimation for propagated segmentations and its application to hippocampal segmentation and brain parcelation. Medical Image Analysis 17(6), 671-684 (Aug 2013)

4. Charon, N., Trouvé, A.: The Varifold Representation of Nonoriented Shapes for Diffeomorphic Registration. SIAM Journal on Imaging Sciences 6(4), 2547-2580 (Jan 2013)

5. Cury, C., Glaunès, J.A., Colliot, O.: Diffeomorphic iterative centroid methods for template estimation on large datasets. In: Nielsen, F. (ed.) Geometric Theory of Information, pp. 273-299. Signals and Communication Technology, Springer International Publishing (2014)

6. Datar, M., Muralidharan, P., Kumar, A., Gouttard, S., Piven, J., Gerig, G., Whitaker, R., Fletcher, P.T.: Mixed-Effects Shape Models for Estimating Longitudinal Changes in Anatomy. In: Hutchison, D., Kanade, T., Kittler, J., Kleinberg, J.M., Mattern, F., Mitchell, J.C., Naor, M., Nierstrasz, O., Pandu Rangan, C., Steffen, B., Sudan, M., Terzopoulos, D., Tygar, D., Vardi, M.Y., Weikum, G., Durrleman, S., Fletcher, T., Gerig, G., Niethammer, M. (eds.) Spatio-temporal Image Analysis for Longitudinal and Time-Series Image Data, vol. 7570, pp. 76-87. Springer Berlin Heidelberg, Berlin, Heidelberg (2012)

7. Durrleman, S., Allassonnire, S., Joshi, S.: Sparse Adaptive Parameterization of Variability in Image Ensembles. International Journal of Computer Vision 101(1), 161-183 (Jan 2013)

8. Durrleman, S., Pennec, X., Trouvé, A., Braga, J., Gerig, G., Ayache, N.: Toward a comprehensive framework for the spatiotemporal statistical analysis of longitudinal shape datas. International Journal of Computer Vision 103(1), 22-59 (May 2013)

9. Durrleman, S., Prastawa, M., Charon, N., Korenberg, J.R., Joshi, S., Gerig, G., Trouvé, A.: Morphometry of anatomical shape complexes with dense deformations and sparse parameters. NeuroImage 101(0), $35-49$ (Nov 2014)

10. Durrleman, S., Prastawa, M., Gerig, G., Joshi, S.: Optimal Data-Driven Sparse Parameterization of Diffeomorphisms for Population Analysis. In: Hutchison, D., Kanade, T., Kittler, J., Kleinberg, J.M., Mattern, F., Mitchell, J.C., Naor, M., Nierstrasz, O., Pandu Rangan, C., Steffen, B., Sudan, M., Terzopoulos, D., Tygar, D., Vardi, M.Y., Weikum, G., Szkely, G., Hahn, H.K. (eds.) Information Processing in Medical Imaging, vol. 6801, pp. 123-134. Springer Berlin Heidelberg, Berlin, Heidelberg (2011)

11. Fishbaugh, J., Prastawa, M., Gerig, G., Durrleman, S.: Geodesic Shape Regression in the Framework of Currents. In: Gee, J.C., Joshi, S., Pohl, K.M., Wells, W.M., Zllei, L. (eds.) Information Processing in Medical Imaging, pp. 718-729. No. 7917 in Lecture Notes in Computer Science, Springer Berlin Heidelberg (Jun 2013)

12. Glaunès, J., Qiu, A., Miller, M.I., Younes, L.: Large Deformation Diffeomorphic Metric Curve Mapping. International Journal of Computer Vision 80(3), 317-336 (Mar 2008)

13. Kheyfets, A., Miller, W.A., Newton, G.A.: Schild's Ladder Parallel Transport Procedure for an Arbitrary Connection. International Journal of Theoretical Physics 39(12), 2891-2898 (2000)

14. Lorenzi, M., Ayache, N., Frisoni, G., Pennec, X., et al.: $4 \mathrm{~d}$ registration of serial brain's mr images: a robust measure of changes applied to alzheimer's disease. In: 
MICCAI Workshop, Challenge on Computer-Aided Diagnosis of Dementia Based on Structural MRI Data (2010)

15. Lorenzi, M., Pennec, X.: Efficient Parallel Transport of Deformations in Time Series of Images: From Schilds to Pole Ladder. Journal of Mathematical Imaging and Vision 50(1-2), 5-17 (Oct 2013)

16. Lorenzi, M., Pennec, X., Frisoni, G.B., Ayache, N.: Disentangling normal aging from Alzheimer's disease in structural magnetic resonance images. Neurobiology of Aging 36, S42-S52 (Jan 2015)

17. Modat, M., Cash, D.M., Daga, P., Winston, G.P., Duncan, J.S., Ourselin, S.: Global image registration using a symmetric block-matching approach. Journal of Medical Imaging 1(2), 024003-024003 (Jul 2014)

18. Pizer, S.M., Fletcher, P.T., Joshi, S., Thall, A., Chen, J.Z., Fridman, Y., Fritsch, D.S., Gash, A.G., Glotzer, J.M., Jiroutek, M.R., Lu, C., Muller, K.E., Tracton, G., Yushkevich, P., Chaney, E.L.: Deformable M-reps for 3D medical image segmentations. International Journal of Computer Vision 55(2-3), 85-106 (2003)

19. Rohrer, J.D., Nicholas, J.M., Cash, D.M., van Swieten, J., Dopper, E., Jiskoot, L., van Minkelen, R., Serge A Rombouts, M.J.C., Clegg, S., Espak, M., Mead, S., Thomas, D.L., Vita, E.D., et al.: Presymptomatic cognitive and neuroanatomical changes in genetic frontotemporal dementia in the Genetic Frontotemporal dementia Initiative(GENFI) study: a cross-sectional analysis. The Lancet Neurology 14(3), 253-262 (Mar 2015)

20. Rothman, K.J.: No adjustments are needed for multiple comparisons. Epidemiology (Cambridge, Mass.) 1(1), 43-46 (Jan 1990)

21. Routier, A., Gori, P., Fouquier, A.B.G., Lecomte, S., Colliot, O., Durrleman, S.: Evaluation of morphometric descriptors of deep brain structures for the automatic classification of patients with Alzheimer's disease, mild cognitive impairment and elderly controls. In: MICCAI Workshop, Challenge on Computer-Aided Diagnosis of Dementia Based on Structural MRI Data (Sep 2014)

22. Schölkopf, B., Smola, A., Müller, K.R.: Nonlinear component analysis as a kernel eigenvalue problem. Neural Computation 10(5), 1299-1319 (Jul 1998)

23. Styner, M., Oguz, I., Xu, S., Brechbühler, C., Pantazis, D., Levitt, J.J., Shenton, M.E., Gerig, G.: Framework for the statistical shape analysis of brain structures using SPHARM-PDM. The Insight Journal 1071, 242-250 (2006)

24. Trouvé, A.: Diffeomorphisms groups and pattern matching in image analysis. International Journal of Computer Vision 28(3), 213-221 (1998)

25. Vaillant, M., Glaunès, J.: Surface matching via currents. In: Christensen, G.E. Sonka, M. (eds.) Information Processing in Medical Imaging. Lecture Notes in Computer Science, vol. 3565, pp. 381-392. Springer Berlin Heidelberg (2005)

26. Younes, L.: Jacobi fields in groups of diffeomorphisms and applications. Quarterly of applied mathematics 65, 113-134 (2007) 